\title{
Nondefoliating and Defoliating Strains from Cotton Correlate with Races 1 and 2 of Verticillium dahliae
}

Xiao-Ping Hu, State Key Laboratory of Crop Stress Biology for Arid Areas and College of Plant Protection, Northwest A\&F University, Yangling 712100, China; Suraj Gurung and Dylan P. G. Short, Department of Plant Pathology, and German V. Sandoya, The Genome Center and Department of Plant Science, University of California-Davis, Salinas 93905; Wen-Jing Shang, State Key Laboratory of Crop Stress Biology for Arid Areas and College of Plant Protection, Northwest A\&F University; Ryan J. Hayes, United States Department of AgricultureAgricultural Research Service, Salinas, CA; R. Michael Davis, Department of Plant Pathology, University of California, Davis 95616; and Krishna V. Subbarao, Department of Plant Pathology, University of California-Davis, Salinas

\begin{abstract}
Hu, X.-P., Gurung, S., Short, D. P. G., Sandoya, G. V., Shang, W.-J., Hayes, R. J., Davis, R. M., and Subbarao, K. V. 2015. Nondefoliating and defoliating strains from cotton correlate with races 1 and 2 of Verticillium dahliae. Plant Dis. 99:1713-1720.

Verticillium wilt, caused by Verticillium dahliae, is an important disease of cotton worldwide. Isolates of $V$. dahliae can be characterized as race 1 or race 2 based on the responses of differential cultivars of tomato and lettuce, or as defoliating or nondefoliating based on symptom expression in cotton. To investigate the frequency and distribution of races and defoliation phenotypes of cotton-associated $V$. dahliae, 317 isolates from China, Israel, Turkey, and the United States were tested by polymerase chain reaction (PCR) using defoliating, nondefoliating, and race 1- and race 2-specific primers DF/DR, NDF/NDR, VdAve1F/VdAve1R, and VdR2F/VdR2R, respectively. Of the total, $97.2 \%$ of isolates genotyped as defoliating were also characterized as race 2 , while $90.8 \%$ of isolates genotyped as nondefoliating were also genotyped as race 1 . To verify these results, three cotton cultivars-'FM 2484B2F' (highly resistant),

'98M-2983' (highly susceptible), and 'CA4002' (partially resistant)—used as differentials were each inoculated with 10 isolates characterized by PCR six defoliating/race 2 strains (GH1005, GH1021, HN, XJ2008, XJ592, and reference strain Ls17) and four nondefoliating/race 1 strains (GH1015, GH1016, GH1020, and reference strain Ls16). All defoliating/race 2 isolates except for Ls 17 caused defoliation on 98M-2983 and CA4002. Isolate Ls17 caused defoliation on 98M-2983 only. The nondefoliating/race 1 isolates caused Verticillium wilt symptoms devoid of defoliation on 98M-2983. The greenhouse assays confirmed the molecular identification of race and defoliation phenotype. Although the existence of races has not been previously established among $V$. dahliae isolates from cotton, the longestablished nondefoliating and defoliating population structure corresponded with $V$. dahliae races 1 and 2, respectively.
\end{abstract}

Verticillium dahliae Kleb. is a widely distributed soilborne pathogen that causes vascular wilt in more than 400 plant species, including high-value annual and perennial crops and landscape, fruit, and ornamental trees and shrubs (Bhat and Subbarao 1999; Inderbitzin and Subbarao 2014; Pegg and Brady 2002). Verticillium wilt results in billions of dollars in annual losses worldwide (Klosterman et al. 2009; Pegg and Brady 2002). For example, crop losses may reach 30, 50, and $100 \%$ for cotton (Friebertshauser and DeVay 1982), potato (Klosterman et al. 2009), and lettuce (Subbarao et al. 1997), respectively. The pathogen can survive as microsclerotia for at least 14 years in soil (Wilhelm 1955) and, thus, it is difficult to eliminate the pathogen from soil and manage the disease. The host range of $V$. dahliae continues to expand as new hosts are identified, as documented in recent years (Lu et al. 2013; Subbarao et al. 1997).

Cotton (Gossypium spp.) is currently the most cultivated fiber crop worldwide. Of the four cultivated species-Gossypium hirsutum L. (upland cotton), G. barbadense (sea-island cotton), G. arboretum, and $G$. herbaceum-G. hirsutum provides more than $95 \%$ of the annual cotton production worldwide (Chen et al. 2007). Verticillium wilt of $G$. hirsutum caused by $V$. dahliae is one of the major

Corresponding author: K. V. Subbarao; E-mail: kvsubbarao@ucdavis.edu.

X. P. Hu and S. Gurung contributed equally to this study.

*The $\boldsymbol{e}$-Xtra logo stands for "electronic extra" and indicates that one supplementary table is published online.

Accepted for publication 1 May 2015.

Modified: 15 June 2017.

http://dx.doi.org/10.1094/PDIS-03-15-0261-RE

(C) 2015 The American Phytopathological Society constraints in most cotton production areas of the world (Bell 1992; Friebertshauser and DeVay 1982).

Variation in virulence or aggressiveness has previously been reported among $V$. dahliae isolates from tomato, potato, lettuce, olive, and cotton (Grogan et al. 1979; Schnathorst and Mathre 1966; Schnathorst and Sibbett 1971; Tjamos 1981; Vallad et al. 2006). Based on the virulence assays on differential lettuce ('Salinas' and 'La Brillante') (Vallad et al. 2006) and tomato ('Early Pak' and 'Beef Steak') (Maruthachalam et al. 2010) cultivars, V. dahliae strains have been characterized as races 1 and 2 . Race 1 is defined by the presence of a gene that encodes a virulence factor (Ave1). In tomato, race 1-resistant plants are defined by the presence of the immune receptor-encoding gene Vel (de Jonge et al. 2012; Kawchuk et al. 2001). Race 2 isolates of $V$. dahliae lack Avel and, thus, are not recognized by plants with the $V e$ gene, resulting in disease (de Jonge et al. 2012; Short et al. 2014). Resistance to Verticillium wilt and race specificity in other crops is often hypothesized to be determined by $V e$-like genes. Homologs of $V e l$ exist in Lactuca sativa that map to the same genomic location of $V r l$, a gene conferring resistance to race $1 \mathrm{~V}$. dahliae isolates (Hayes et al. 2011). Other $V e$ homologs have been cloned from Solanum licopersicoides (Chai et al. 2003), S. torvum (Fei et al. 2004), Mentha longifolia (Vining et al. 2007), G. hirsutum (Gao et al. 2011), and G. barbadense (Zhang et al. 2011). A two-gene model system found in S. tuberosum seems to offer a level of protection to potato similar to that conferred by the $V e$ gene to tomato (Jansky et al. 2004). In tomato, MKK2 and NDR1 have been posited as genetic requirements needed in addition to $\mathrm{Vel}$ for resistance against race 1 of $V$. dahliae (Fradin et al. 2009).

Schnathorst and Mathre (1966) first demonstrated differential virulence among $V$. dahliae isolates from cotton in California, and classified them as either defoliating or nondefoliating pathotypes based on the symptoms observed on inoculated plants. Isolates that caused complete defoliation of cotton were called 
defoliating and those that only caused wilt without defoliation were called nondefoliating. The defoliating isolate T-1 (later renamed as T9) was highly virulent on 'Tanguis' ( $G$. barbadense), 'Acala 4-42' (G. hirsutum), and 'Delta Pine 15' (DPL) (G. hirsutum) cotton, whereas the nondefoliating isolate SS4 was mildly virulent on these cultivars (Schnathorst and Mathre 1966). Pérez-Artés et al. (2000) developed specific polymerase chain reaction (PCR) primer pairs to identify defoliating and nondefoliating strains of $V$. dahliae based on random amplified polymorphic DNA. In the Commonwealth of Independent States, five races $(0,1,2,3$, and 4) were recognized based on virulence on cotton cultivars (Popov et al. 1972; Portenko and Kas'yanenko 1987). Race 1 was avirulent on all cultivars tested, races 2 and 3 defoliate 'Tashkent' and G. hirsutum Acala 4-42, while races 1 and 4 are nondefoliating types. Hitherto, the defoliating type has been reported from most cotton-growing regions in the Americas (Daayf et al. 1995; Schnathorst and Mathre 1966), China (Daayf et al. 1995; Xia et al. 1998), France (Daayf et al. 1995), Spain (Bejarano-Alcázar et al. 1995), and Israel (Korolev et al. 2008).

$V$. dahliae isolates from cotton have also been classified based on vegetative compatibility groups (Puhalla and Hummel 1983), lysozymes (Okoli et al. 1993), molecular markers (Carder and Barbara 1994; Pérez-Artés et al. 2000; Ramsay et al. 1996), and immunochemical techniques (Nachmias et al. 1985). Isolates of $V$. dahliae from tomato (de Jonge et al. 2012) and other crops (Gurung et al. 2014; Short et al. 2014) may be rapidly characterized genetically as race 1 and race 2 with specific PCR primer pairs because those that carry Ave1 are considered race 1, while those that do not are considered race 2.

A few studies have explored the role of Gbvel in disease resistance in the context of defoliating and nondefoliating isolates of $V$. dahliae. Gbvel was previously reported to confer resistance to transgenic Arabidopsis and cotton against both defoliating (V991) and nondefoliating (BP2) strains of $V$. dahliae from cotton (Zhang et al. 2012). However, in a separate study, transgenic Arabidopsis lines containing Vel and Gbvel showed improved disease resistance only to nondefoliating strains of $V$. dahliae (Fradin et al. 2011; Zhang et al. 2011). Similarly, Gao et al. (2011) silenced GhVe1, GhNDR1, and GhMKK2 in cotton, which resulted in increased susceptibility to $V$. dahliae, suggesting that race 1 of $V$. dahliae is probably the nondefoliating type in cotton. To our knowledge, the putative relationship between defoliation phenotype and race in $V$. dahliae isolates collected from cotton or olive has not been thoroughly investigated. This information will be very helpful for cotton breeders developing cotton cultivars with resistance to $V$. dahliae.

The objectives of this study were to (i) determine the race and defoliation phenotype composition of a global sample of $V$. dahliae from cotton using PCR assays and (ii) assess the correlation between nondefoliating or defoliating phenotypes and race 1 or race $2 \mathrm{~V}$. dahliae isolates from cotton.

\section{Materials and Methods}

Strains. In all, $317 \mathrm{~V}$. dahliae isolates from cotton, lettuce, and tomato were included in this study (Supplementary Table S1). The collection comprised cotton isolates from China $(n=262)$, Israel $(n=1)$, Turkey $(n=30)$, and the United States $(n=24)$. Strains Ls16 (race 1) and Ls17 (race 2) from lettuce, Le1087 (race 1) supplied by the Campbell Soup Company (Davis, CA), and Le1811 (race 2) from tomato were used as reference strains (Maruthachalam et al. 2010, Short et al. 2014). All isolates were obtained from symptomatic plants collected in commercial fields. They were morphologically identified as Verticillium spp. based on features such as microsclerotia and verticillate conidiophores on potato dextrose agar (PDA) and were confirmed as $V$. dahliae sensu stricto using PCR as previously described by Inderbitzin and Subbarao (2014).

PCR primers. The primers used to identify defoliating strains of $V$. dahliae were DF (CATGTTGCTCTGTTGACTGG) and DR (GACACGGTATCTTTGCTGAA), and the primers used for identifying nondefoliating strains of $V$. dahliae were NDF (CAGGGGATACTGGTACGAGACG) and NDR (ATGAGTATTGCCGATAAGAACA) (Pérez-Artés et al. 2000). The primers VdAve1F (AAGGGGTCTTGCTAGGATGG) and VdAve1R (TGAAACACTT GTCCTCTTGCT) (de Jonge et al. 2012) were used to identify $V$. dahliae race 1 (Avirulence on $\mathrm{Vel}$ ), whereas the primer pair $\mathrm{VdR} 2 \mathrm{~F}$ (ACTTAACGAAAGCATGCGC) and VdR2R (CTTGACTTG CCGGCTCC) was used to identify $V$. dahliae race 2 (Short et al. 2014). Primers for $V$. dahliae species identification were previously described by Inderbitzin et al. (2013). All primers were synthesized by Integrated DNA Technologies Inc. (Coralville, IN). All isolates were tested with DF/DR, NDF/NDR, VdAve1F/VdAve1R, and VdR2F/VdR2R primer pairs.

DNA extraction and PCR. V. dahliae isolates were single-spored on PDA plates, transferred into $150-\mathrm{ml}$ glass flasks with $50 \mathrm{ml}$ of potato dextrose broth (PDB), and incubated on laboratory benches $\left(23 \pm 1^{\circ} \mathrm{C}\right)$ for 7 to 10 days. Mycelia were harvested from PDB and washed thoroughly with double-sterilized distilled water, dried with paper towels, and stored at $-80^{\circ} \mathrm{C}$ until use. Mycelia (approximately $50 \mathrm{mg}$ ) were ground to a fine powder in liquid nitrogen with a mortar and pestle, transferred into a 2.0-ml safelock microcentrifuge tube together with $3-\mathrm{mm}$ tungsten carbide beads and $400 \mu \mathrm{l}$ of lysis buffer, and disrupted for $30 \mathrm{~s}$ at full speed with a Qiagen TissueLyser. Other procedures were the same as described in the protocol of the DNeasy Plant Mini Kit (Qiagen). DNA samples were adjusted to a final concentration of $10 \mathrm{ng} / \mu \mathrm{l}$ with a NanoDrop 1000 spectrophotometer and kept at $4{ }^{\circ} \mathrm{C}$ until use. All PCR were separately performed using four primer pairs (DF/DR, NDF/NDR, VdAve1F/VdAve1R, and VdR2F/VdR2R) as previously described with a PTC-100 thermocycler (MJ Research) (de Jonge et al. 2012; Pérez-Artés et al. 2000; Short et al. 2014). All PCR amplifications were performed in $25-\mu 1$ reaction volumes containing $10 \mu l$ of sterile distilled water, $12 \mu \mathrm{l}$ of $2 \times$ GoTaq master mix (Promega Corp.), $1 \mu \mathrm{l}$ of genomic DNA at $10 \mathrm{ng} / \mu \mathrm{l}$, and $1 \mu \mathrm{l}$ of each primer at a concentration of $10 \mu \mathrm{M}$. The annealing temperatures of defoliating, nondefoliating, and race 1 - and race 2 -specific primers were $61,58,62$, and $64^{\circ} \mathrm{C}$, respectively. Each PCR was stained with $8 \mu \mathrm{l}$ of SyberGold (Invitrogen Life Technologies), and aliquots were loaded in $1.5 \%(\mathrm{wt} / \mathrm{vol})$ agarose gels and run for $120 \mathrm{~min}$ at $75 \mathrm{~V}$ in $0.5 \%$ Tris-borate-EDTA buffer. A transilluminator (Ultra-Violet Products, Ltd.) was used for PCR product visualization, and a 100-bp DNA ladder (Invitrogen Life Technologies) was included on each gel.

Seedling production and virulence assays. Eight cotton isolates were used in virulence assays in the greenhouse to determine nondefoliating and defoliating phenotypes: three isolates with Ave1- and nondefoliating-specific PCR bands (GH1015, GH1016, and GH1020) and five isolates with race 2 and defoliating-specific PCR bands (GH1005, GH1021, HN, XJ2008, and XJ592), which were previously confirmed as defoliating strains (Hu et al. 2014; Maruthachalam et al. 2010; Zhu et al. 2012). In addition, the race 1 and race 2 reference strains Ls16 and Ls17, originally isolated from lettuce, were also used in virulence assays as controls.

Three differential cultivars of cotton were used: 'FM 2484B2F' (highly resistant to Verticillium wilt) (K. V. Subbarao and X.-P. $\mathrm{Hu}$, unpublished data), supplied by Bayer CropScience; '98M2983' (highly susceptible to Verticillium wilt); and 'CA4002' (partially resistant to Verticillium wilt) (Dever et al. 2013), supplied by the Cotton Genetics Research Unit, United States Department of AgricultureAgricultural Research Service. Seed of each cultivar were planted into 50-well trays containing Sunshine Professional Growing Mix Number 5 (SUNGRO Horticulture) and grown in a greenhouse until the two-true-leaf stage (approximately 4 weeks). Seedling roots were then uprooted, rinsed free of soil under tap water, and dipped in a conidial suspension containing $1 \times 10^{7}$ conidia $/ \mathrm{ml}$ for $15 \mathrm{~min}$. Seedlings dipped in sterile distilled water for $15 \mathrm{~min}$ were used as noninoculated controls. All seedlings were transplanted into 0.5liter plastifoam-Hot-Cups (Amerifoods) filled with a pasteurized sand/potting soil mixture $(2: 1, \mathrm{vol} / \mathrm{vol})$ and maintained for an 
additional 8 weeks in a greenhouse with lighting (16-h photoperiod) from June to September 2014. Greenhouse temperatures, on average, were maintained at $24 \pm 5^{\circ} \mathrm{C}$ during daytime and $12 \pm 5^{\circ} \mathrm{C}$ at night. For each $V$. dahliae isolate, seven plants were inoculated per experiment (replicates) and the whole experiment was conducted twice.

Defoliation and nondefoliation of leaves from three cotton cultivars were recorded 60 days after inoculation. Additionally, the Verticillium wilt severity of each plant was evaluated according to vascular discoloration in inoculated and control plants with a 0 -to- 4 ordinal scale, where $0=$ no vascular discoloration; $1=1$ to $25 \%$ of the vascular tissue exhibiting discoloration; and $2=26$ to $50,3=51$ to 75 , and $4=76$ to $100 \%$ discoloration in the presence of typical foliar symptoms of Verticillium wilt. The plant height was also measured just before disease severity assessment. Symptomatic vascular tissues were surface disinfested using distilled water and 5\% bleach, dissected, and plated on NP-10 medium (Kabir et al. 2004). Following 2 weeks of incubation at ambient temperature, plates were examined under a stereomicroscope for the presence of morphological features and cultural characteristics typical of $V$. dahliae, including microsclerotia, verticillate conidiophores, and hyaline conidia.

Statistical analysis of virulence data. Nonparametric data analyses (Shah and Madden 2004) were performed using SAS (version 9.3; SAS Institute), as described previously (Vallad et al. 2006), because the Verticillium wilt severity of cotton was evaluated using an ordinal disease rating scale. The experiment was set as a split-plot design, in which strains were considered whole plots and cotton cultivars were considered subplots. The two experiments were used as additional factor. A null hypothesis that treatments were different from zero was tested using the analysis of variance (ANOVA)-type statistic of the ranked method utilizing PROC MIXED, as described by Shah and Madden (2004). The relative marginal effects (RME) and $95 \%$ confidence intervals for each treatment were calculated using the LD_CI and F1_LD_F1 macros written with PROC IML of the SAS Institute. Orthogonal linear contrasts were constructed to compare the cotton isolates against the race 1 and race 2 lettuce isolates Ls 16 and Ls17, respectively, and probabilities associated with these contrasts were computed.

For plant height, ANOVA was run using PROC GLM in SAS. The data were analyzed separately for each cultivar. Means were separated using Fisher's protected least significant difference test at $95 \%$ probability. In addition, PROC CORR was used to calculate the correlations between plant height and disease severity for each cultivar.

\section{Results}

Assessment of correlation between nondefoliating or defoliating phenotypes and races. In total, 317 cotton isolates from China, Israel, Turkey, and the United States; strains Ls16 (race 1) and Ls17 (race 2) from lettuce; and Le1087 (race 1) and Le1811 (race 2) from tomato were identified as $V$. dahliae using the species-specific PCR assay. The primer pairs DF/DR, NDF/NDR, VdAve1F/VdAve1R, and VdR2F/VdR2R were employed to determine the defoliation type and race of all isolates with PCR, based on the presence of 550-bp (defoliating), 1,500-bp (nondefoliating), 900-bp (race 1), and 256-bp (race 2) amplicons (Fig. 1). Of the 317 isolates, 181 were positively amplified using the DF/DR primer pair and designated as defoliating type, whereas 130 isolates were amplified using the NDF/NDR primer pair and designated as nondefoliating type. Nine of the isolates could not be amplified with either of the primers. Similarly, of the same 317 isolates, 118 were amplified using the race 1 -specific primer pair (VdAve1F/VdAve1R) and designated as race 1, whereas 194 isolates were successfully amplified using the race 2-specific primer pair (VdR2F/VdR2R) and designated as race 2. Eight isolates could not be amplified with either race-specific primer. Interestingly, $97.2 \%$ of strains with defoliating type bands correlated with race 2 , while $90.8 \%$ of strains with nondefoliating type bands correlated with race 1.

Validation of defoliating and nondefoliating types in greenhouse experiments. The typical symptoms of Verticillium wilt were visible on 98M-2983 and CA4002 at approximately 20 and 25 days post inoculation, respectively. The first defoliation symptoms on 98M-2983 and CA4002 appeared at approximately 30 and 36 days, respectively, following inoculations with XJ592. In 98M-2983, defoliation occurred following inoculations with GH1005, GH1021, HN, XJ2008, XJ592, and Ls17, whereas the plants inoculated with GH1020, GH1016, GH1015, and Ls 16 showed typical Verticillium wilt but leaves remained intact until 60 days postinoculation (Fig. 2). These results were consistent with the defoliating and nondefoliating type results detected through PCR assays. The defoliation symptom was also observed on the partially resistant cotton CA4002 plants inoculated with V. dahliae isolates XJ592, XJ2008, GH1005, GH1021, and HN (Fig. 3). However, wilt symptoms on plants inoculated with $V$. dahliae isolate Ls 17 were not observed. The leaf symptom on cotton 98M-2983 inoculated with $V$. dahliae isolate Ls17 and Ls16 appeared only at approximately 50 and 54 days postinoculation, respectively. Lettuce strains Ls 16 and Ls17 did not cause symptoms on FM 2484B2F and CA4002 in this study. The highly resistant cotton FM 2484B2F remained resistant to all inoculated isolates.

Comparison of virulence on host differentials. The heights of FM 2484B2F plants inoculated with all $10 \mathrm{~V}$. dahliae isolates were similar (Fig. 4A). However, the plants of 98M-2983 inoculated with the $10 \mathrm{~V}$. dahliae isolates were stunted (Fig. 4B). In contrast, only the

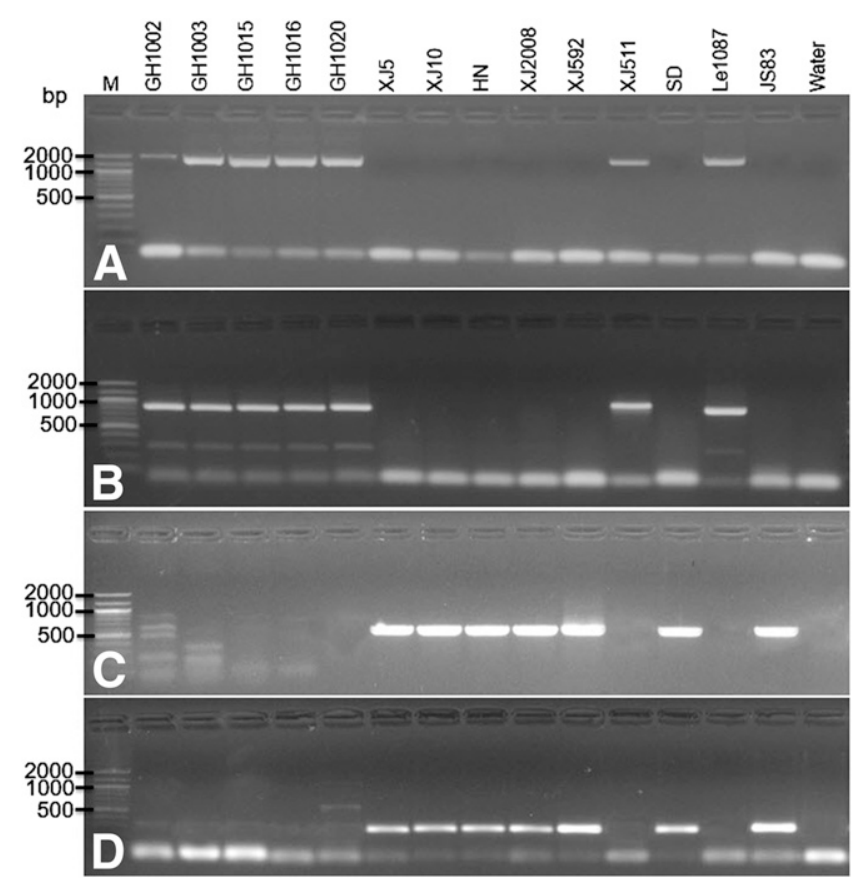

Fig. 1. Agarose gel pattern of polymerase chain reaction amplicons generated with defoliating, nondefoliating, and race 1- and race 2-specific primer pairs of Verticillium dahliae. A, Amplicons generated with the nondefoliating-specific primers NDF and NDR. B, Amplicons generated with the race 1-specific primers VdAve1F and VdAve1R. C, Amplicons generated with the defoliatingspecific primers DF and DR. D, Amplicons generated with the race 2-specific primers $\mathrm{VdR} 2 \mathrm{~F}$ and $\mathrm{VdR} 2 \mathrm{R}$. The $1.5 \%(\mathrm{wt} / \mathrm{vol})$ agarose gel was used. Lane $M=2,000-b p$ DNA ladder used as a size marker. Strains $\mathrm{GH} 1002, \mathrm{GH} 1003$, GH1015, GH1016, GH1020, and GH1021 collected from cotton, California; strain HN collected from cotton, Henan, China; strains XJ2008 and XJ592 collected from cotton, Xinjiang, China; strains Ls16 and Ls17 collected from lettuce, California; strains Le1811 and Le1087 collected from tomato, California. Ls16 and Le1087 were used as positive control for race 1. Ls17 and Le1811 were used as positive control for race 2. Sterile distilled water was used as a negative control. 
plants of CA4002 inoculated with XJ592 and XJ2008 were stunted (Fig. 4C).

A wide variation in the virulence of $V$. dahliae on the three differential cotton cultivars was observed. There were significant effects both of strain and cultivar on the Verticillium wilt $(P<$ $0.0001)$. In addition, there was a significant interaction of strain and cultivar $(P=0.0472)$, suggesting that wilt severity on a cultivar was dependent on the $V$. dahliae isolate used for inoculation (Table 1).

The median disease ratings, mean ranks, variances, RME, and 95\% confidence lower and upper limits of RME are shown in Table 2. Median disease ratings ranged from 0.0 to 1.0 for FM 2484B2F, 3.3 to 5.0 for $98 \mathrm{M}-2983$, and 0.0 to 4.4 for CA4002; RME ranged from 0.15 to $0.48,0.15$ to 0.96 , and 0.15 to 0.80 for the corresponding three cultivars (Table 2). In the resistant FM 2484B2F, isolates from the defoliating strain GH1005, GH1021, HN, XJ2008, and XJ592 caused slightly higher wilt severity than race $1 /$ nondefoliating strains (Fig. $5 \mathrm{~A}$ ). Even though differences in the severity caused by race $1 /$ nondefoliating and race 2 /defoliating strains on the susceptible $98 \mathrm{M}-2983$ were not significant (Fig. 5B), they could be distinguished by the absence or presence of defoliation symptoms. However, in the partially resistant CA4002, the severity caused by the defoliating/race 2 strains was higher than those caused by the nondefoliating/race 1 strains (Fig. 5C).

Linear contrasts of disease severity of each isolate from cotton compared with Ls16 and Ls17 showed that only HN, Ls17, XJ2008, and XJ592 were significantly ( $P=0.0120$ to 0.0001$)$ different from Ls16 but not from Ls17 ( $P=0.0760$ to 0.7460$)$ (Table 3).

\section{Discussion}

Recent findings of a virulence effector (de Jonge et al. 2012) (Avel gene) in several $V$. dahliae race 1 isolates from tomato and lettuce paved the way to elucidate the potential race structure in $V$. dahliae isolates from hosts in which host-pathogen genotype interactions are not as well defined as in tomato and lettuce. In cotton, characterization of $V$. dahliae isolates hitherto was limited to defoliating and nondefoliating phenotypes on inoculated cotton plants and molecular markers. Results from this study clearly suggest that inherent within such characterizations is the presence of a race structure similar to those observed in lettuce and tomato. This was further validated by the pathogenicity assays using differential cotton cultivars. Variation in virulence and aggressiveness in $V$. dahliae from cotton has been previously documented (Joaquim and Rowe 1990; Puhalla and Hummel 1983; Schnathorst and Mathre 1966), similar to the variation observed in $V$. dahliae isolates from other hosts, including tomato, potato, and lettuce, (Grogan et al. 1979; Tjamos 1981; Vallad et al. 2006). Lettuce and tomato are currently the only two crops in which host differentials to delineate races in $V$. dahliae are available (Maruthachalam et al. 2010; Vallad et al. 2006). In tomato, although host resistance against $V$. dahliae was identified several decades ago, the molecular basis of this resistance was understood only recently. Resistance against race 1 is mediated by the presence of an immune receptor-encoding gene ( $\mathrm{Vel}$ and its homologs; de Jonge et al. 2012). V. dahliae race 1 is defined by the presence of a gene that encodes a virulence factor (Ave1). Tomato plants with the $V e$ gene limit the colonization of root tissues by the pathogen, preventing the pathogen from reaching foliar tissues (Heinz et al. 1998).

Nearly $37 \%$ of the $V$. dahliae isolates from cotton included in this study were characterized as race 1 /nondefoliating. The sequences of products amplified from $V$. dahliae cotton strains using VdAve $1 \mathrm{~F}$ and VdAveR primer pairs were $100 \%$ matches with Ave1 gene sequences of Ls16 from lettuce and Le1087 from tomato (data not shown). This was further validated by virulence assays on the highly susceptible 98M-2983. Correspondingly, about $61 \%$ of the $V$. dahliae isolates from cotton were of race $2 /$ defoliating type. The results of the greenhouse experiments and PCR assays clearly suggest that strains of $V$. dahliae from cotton can be categorized as race 1 and race 2 using methods established for lettuce and tomato (Maruthachalam et al. 2010; Vallad et al. 2006). This evolution of $V$. dahliae race 2 populations
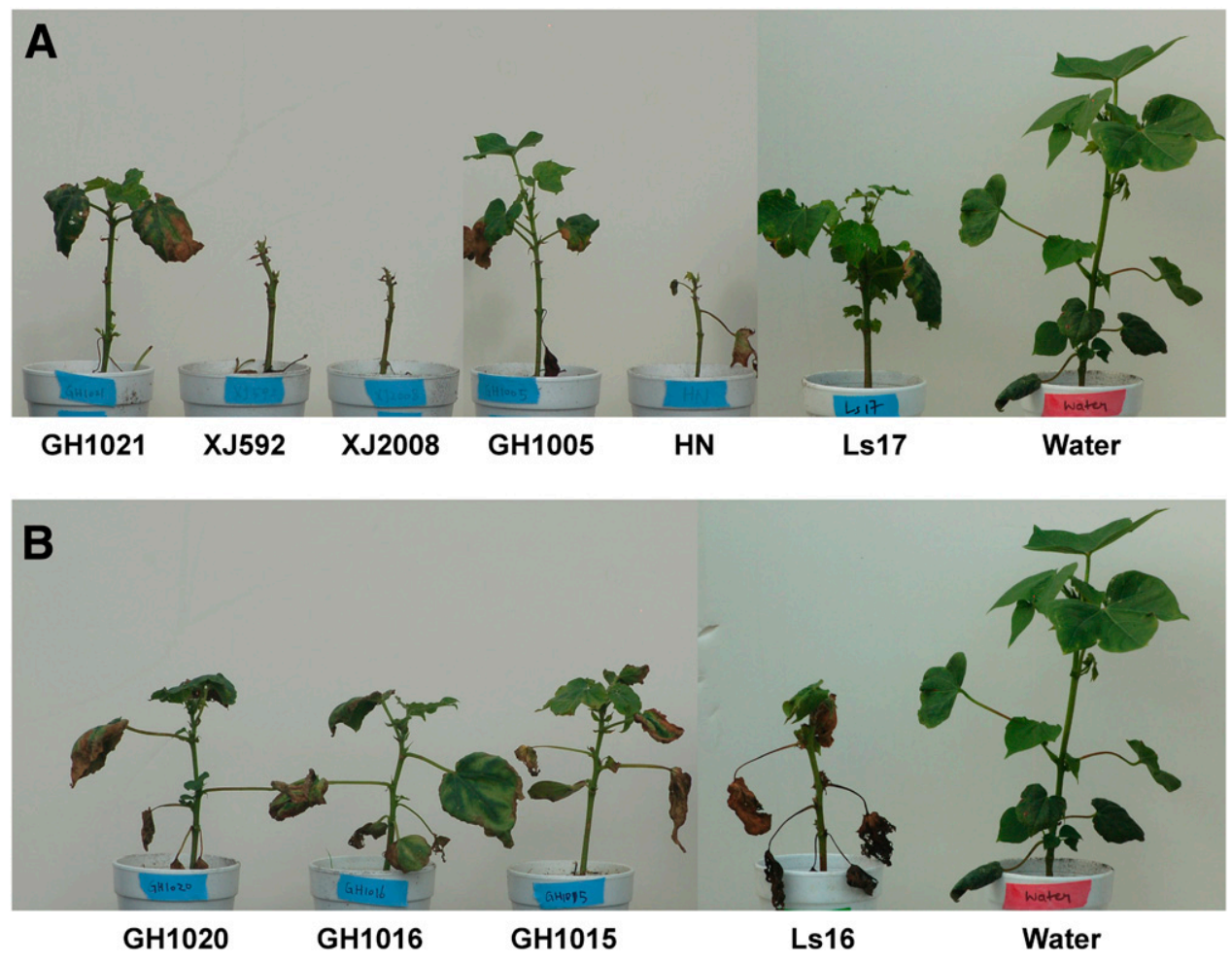

Fig. 2. Differential response of susceptible cotton $98 \mathrm{M}-2983$ at 8 weeks after inoculation with conidial suspension $\left(1 \times 10^{7}\right.$ conidia/ml) of Verticillium dahliae isolates. A, Typical defoliating symptom was apparent among isolates of GH1005, GH1021, HN, XJ2008, XJ592, and Ls17 on susceptible 98M-2983. B, Typical nondefoliating symptom was apparent among isolates of GH1015, GH1016, GH1020, and Ls16 on susceptible cultivar 98M-2983. 

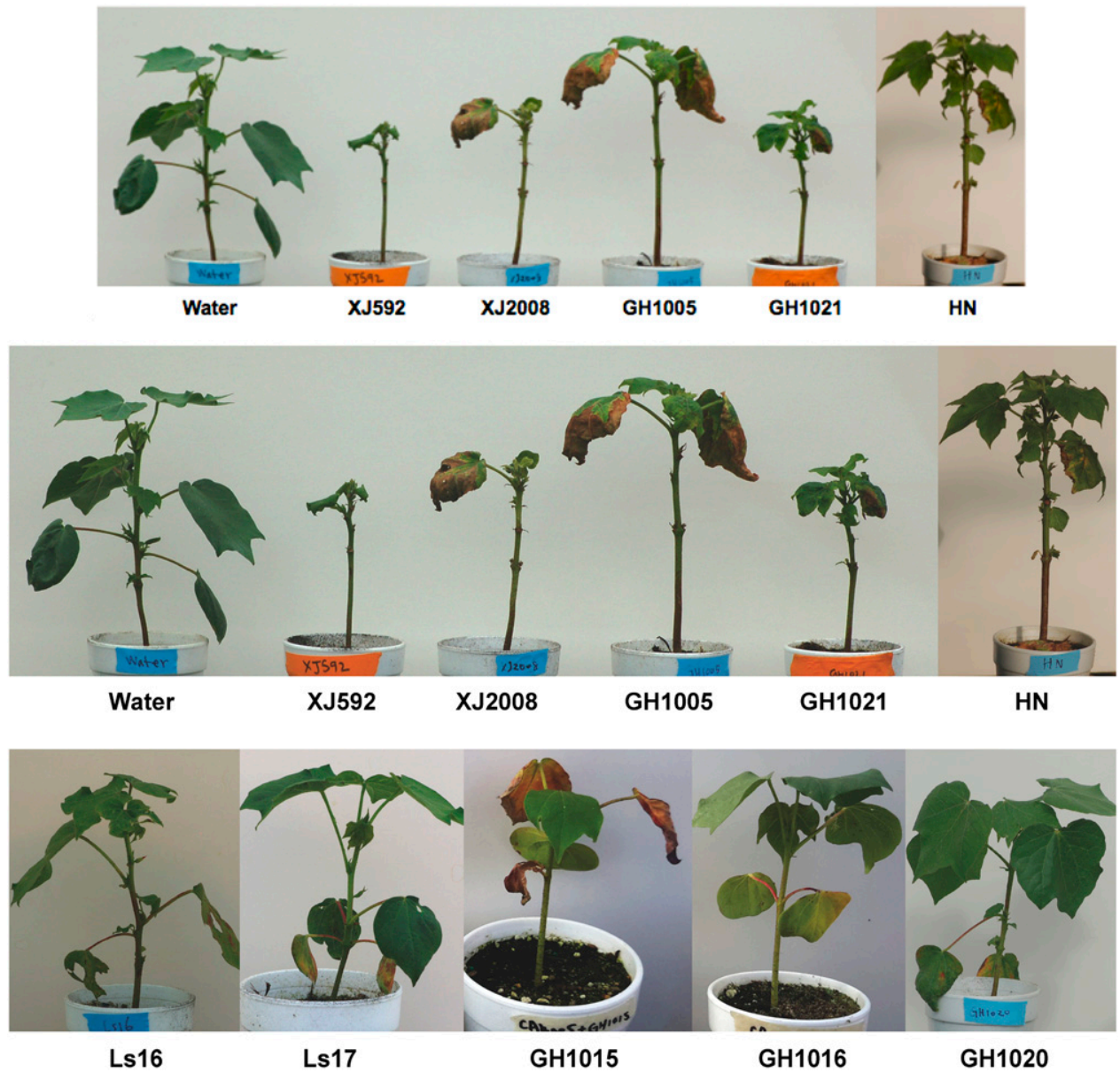

Fig. 3. Differential response of partially resistant cotton CA4002 at 8 weeks after inoculation with conidial suspension $\left(1 \times 10^{7}\right.$ conidia/ml) of Verticillium dahliae isolates. Typical defoliating symptoms were apparent among XJ592, XJ2008, GH1005, GH1021, and HN. No symptom was found in the plants inoculated with Ls16, Ls17, GH1016, and GH1020. Typical nondefoliating symptom was apparent in GH1015. Plant roots dipped in sterile distilled water were used as a negative control.
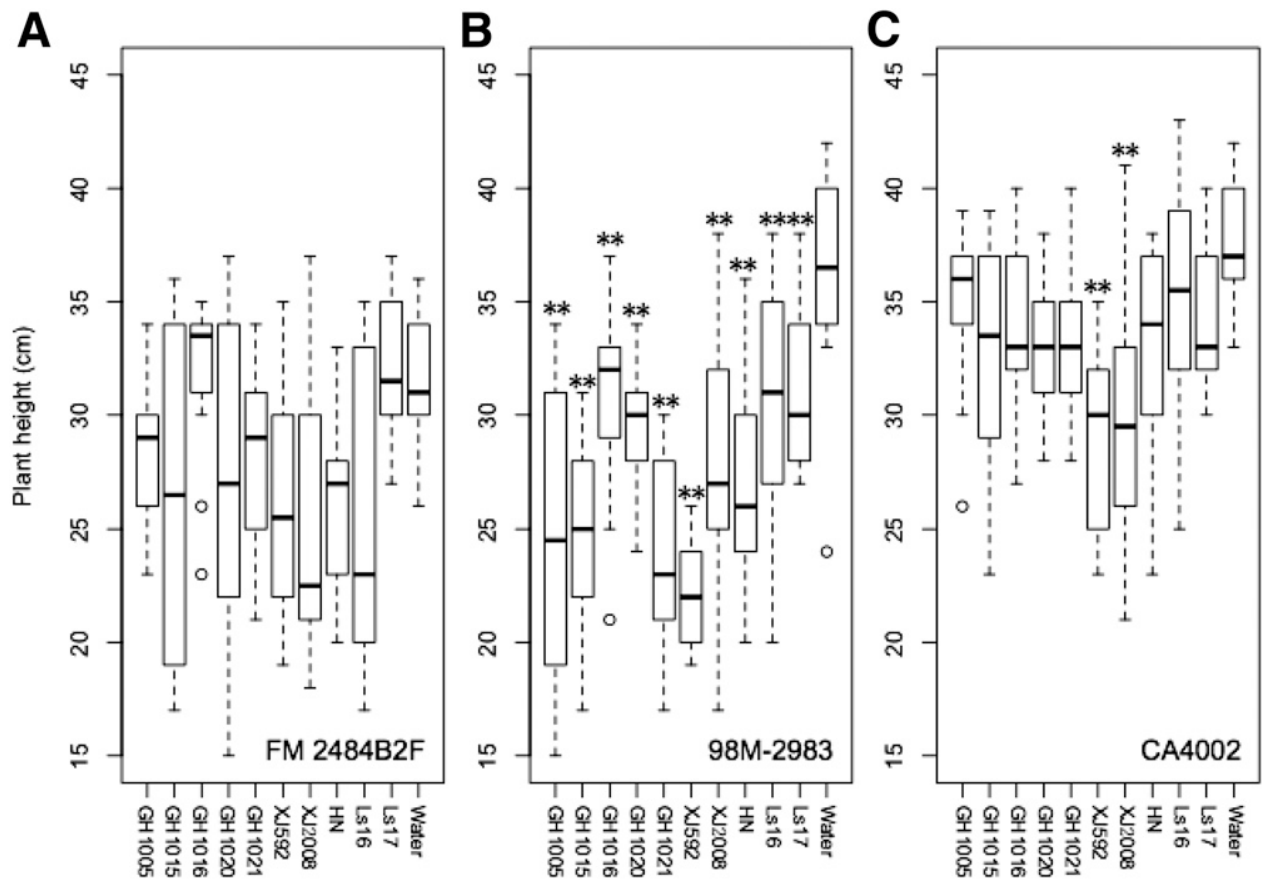

Fig. 4. Plant height of FM 2484B2F, 98M-2983, and CA4002 cotton at 60 days after inoculation with 10 Verticillium dahliae strains. Control plants were inoculated with sterile distilled water. A, No significant difference was observed between the plant height of resistant FM 2484B2F cotton plants inoculated with strains of GH1005, GH1015, GH1016, GH1020, GH1021, XJ592, XJ2008, HN, Ls16, and Ls17 and water $(P>0.05)$. B, Susceptible 98M-2983 cotton plants inoculated with all $10 \mathrm{~V}$. dahliae strains were significantly different from negative control $(P<0.05)$ and were stunted. C, Partially resistant CA4002 cotton inoculated with XJ592 and XJ2008 were significantly different from negative control $(P<0.05)$ and were stunted. 
associated with cotton may also be related to the deployment of commercial cotton cultivars with resistance or tolerance to $V$. dahliae race 1 type. The higher numbers of race 2 over race 1 from the areas sampled could be due to the deployment of race 1-resistant cotton cultivars. In tomato, outbreaks of Verticillium wilt linked to race 2 of $V$. dahliae became common soon after tomato cultivars with the $V e$ gene (Alexander 1962) were deployed in California (Grogan et al. 1979) and subsequently worldwide (Pegg and Brady 2002). Similarly, 311 of $329 \mathrm{~V}$. dahliae isolates $(94.52 \%)$ collected from lettuce during 1995 to 2013 were race 1 (Gurung et al. 2014), which is in line with the fact that commercial lettuce cultivars possessing the race 1 resistance gene $\mathrm{Vrl}$ are not yet available. In due course, with the deployment of race 1 resistance in lettuce, a scenario similar to tomato is expected to occur in this cropping system. Further investigations are needed to elucidate whether cotton cultivars planted in the past several decades in China or elsewhere contain Vel homologs and the extent of their cultivation.
There are several reports of $V$. dahliae from cotton being differentiated into two defoliating and nondefoliating types (BejaranoAlcázar et al. 1995; Friebertshauser and DeVay 1982; Korolev et al. 2008; Pérez-Artés et al. 2000; Schnathorst and Mathre 1966). Currently, the genetic basis to explain the existence of the two pathotypes is lacking. However, the current study provides strong evidence that the nondefoliating and defoliating phenotypes from cotton are strongly associated with race 1 and race 2 genotypes, respectively. The nondefoliation or defoliation pathotypes of $V$. dahliae from cotton could be easily identified through race 1 - or race 2 -specific primer pairs. However, eight isolates (from Turkey) that were amplified with nondefoliating primers were also amplified with race 2 -specific primers but not with race 1 -specific primers. Similarly, one isolate from China that was amplified with defoliating primers was also amplified with race 1 -specific primers. This shows that the pathotype or race structure in $V$. dahliae isolates collected from cotton is more complex than

Table 1. Test statistics for the effects of cultivar and strains on Verticillium dahliae type wilt of cotton seedlings using nonparametric analysis of PROC MIXED procedure in SAS ${ }^{\mathrm{a}}$

\begin{tabular}{|c|c|c|c|c|c|c|c|c|}
\hline \multirow[b]{2}{*}{ Effect } & \multirow[b]{2}{*}{$\mathbf{D F}_{\mathbf{N}}$} & \multirow[b]{2}{*}{$\mathbf{D F}_{\mathbf{D}}$} & \multirow[b]{2}{*}{$x^{2}$} & \multirow[b]{2}{*}{$P$ value } & \multicolumn{4}{|c|}{ ANOVA $F$} \\
\hline & & & & & DFN & DFD & Value & $P$ value \\
\hline Strain & 11 & 12 & 1003.58 & $<0.0001$ & 7.63 & 14.2 & 11.17 & $<0.0001$ \\
\hline Cultivar & 2 & 24 & 177.44 & $<0.0001$ & 1.94 & 14.2 & 98.75 & $<0.0001$ \\
\hline Strain $\times$ cultivar & 22 & 24 & 514.77 & $<0.0001$ & 11.4 & 14.2 & 2.58 & 0.0472 \\
\hline
\end{tabular}

a ANOVA $=$ analysis of variance, $\mathrm{DF}_{\mathrm{N}}=$ numerator degrees of freedom and $\mathrm{DF}_{\mathrm{D}}=$ denominator degrees of freedom.

Table 2. Relative marginal effects (RME) of disease severity for cotton Verticillium wilt severity ratings in relation to different hosts and Verticillium dahliae strains $^{\mathrm{a}}$

\begin{tabular}{|c|c|c|c|c|c|c|c|c|c|}
\hline Cultivar & Strain & $\begin{array}{c}\text { Median } \\
\text { disease rating }\end{array}$ & $\begin{array}{l}\text { Mean } \\
\text { ranks }\end{array}$ & Variance & RME & $\begin{array}{l}\text { 95\% Confidence } \\
\text { low limit }\end{array}$ & $\begin{array}{l}\text { 95\% Confidence } \\
\text { upper limit }\end{array}$ & $\begin{array}{l}P \text { value compared } \\
\text { with Ls16 }\end{array}$ & $\begin{array}{c}P \text { value compared } \\
\text { with } L s 17\end{array}$ \\
\hline \multirow[t]{11}{*}{ FM2484B2F } & GH1005 & 1.0 & 34.0 & 0.105 & 0.47 & 0.12 & 0.13 & 0.0972 & 0.0022 \\
\hline & GH1015 & 0.2 & 20.3 & 0.296 & 0.27 & 0.16 & 0.26 & 0.3030 & 0.3487 \\
\hline & GH1016 & 0.0 & 11.5 & 0.014 & 0.15 & 0.04 & 0.05 & 1.0000 & 1.0000 \\
\hline & GH1020 & 0.3 & 22.3 & 0.448 & 0.30 & 0.19 & 0.31 & 0.3938 & 0.4203 \\
\hline & GH1021 & 0.8 & 24.8 & 0.758 & 0.34 & 0.24 & 0.37 & 0.4437 & 0.4542 \\
\hline & $\mathrm{HN}$ & 0.9 & 35.0 & 0.023 & 0.48 & 0.06 & 0.06 & 0.1363 & 0.1363 \\
\hline & Ls16 & 0.0 & 11.5 & 0.014 & 0.15 & 0.04 & 0.05 & $\mathrm{NC}$ & 1.0000 \\
\hline & Ls17 & 0.0 & 11.5 & 0.014 & 0.15 & 0.04 & 0.05 & 1.0000 & $\ldots$ \\
\hline & XJ2008 & 0.4 & 27.0 & 0.050 & 0.37 & 0.08 & 0.09 & 0.1158 & $\ldots$ \\
\hline & XJ592 & 0.3 & 22.3 & 0.498 & 0.30 & 0.20 & 0.32 & 0.6024 & 0.6024 \\
\hline & Water & 0.0 & 11.5 & 0.014 & 0.15 & 0.04 & 0.05 & $\ldots$ & $\ldots$ \\
\hline \multirow[t]{11}{*}{$98 \mathrm{M}-2983$} & GH1005 & 4.2 & 61.8 & 0.249 & 0.85 & 0.32 & 0.11 & 0.4680 & 0.5257 \\
\hline & GH1015 & 4.9 & 66.5 & 0.034 & 0.92 & 0.12 & 0.05 & $\mathrm{NC}$ & 0.2657 \\
\hline & GH1016 & 3.5 & 50.0 & 0.277 & 0.69 & 0.24 & 0.16 & 0.2124 & 0.7355 \\
\hline & GH1020 & 3.7 & 55.0 & 0.308 & 0.76 & 0.28 & 0.15 & $\mathrm{NC}$ & 0.7578 \\
\hline & GH1021 & 5.0 & 69.5 & 0.002 & 0.96 & 0.03 & 0.01 & 0.1468 & 0.2363 \\
\hline & $\mathrm{HN}$ & 4.7 & 62.8 & 0.046 & 0.86 & 0.11 & 0.06 & $\mathrm{NC}$ & 0.3944 \\
\hline & Ls16 & 3.7 & 53.8 & 0.228 & 0.74 & 0.23 & 0.14 & $\ldots$ & 0.9202 \\
\hline & Ls17 & 3.4 & 51.8 & 0.538 & 0.71 & 0.34 & 0.20 & 0.9202 & $\ldots$ \\
\hline & XJ2008 & 3.3 & 49.5 & 0.113 & 0.68 & 0.15 & 0.12 & 0.6788 & 0.7823 \\
\hline & XJ592 & 5.0 & 69.5 & 0.002 & 0.96 & 0.03 & 0.01 & 0.1468 & 0.2363 \\
\hline & Water & 0.0 & 11.5 & 0.014 & 0.15 & 0.04 & 0.05 & $\ldots$ & $\ldots$ \\
\hline \multirow[t]{11}{*}{ CA4002 } & GH1005 & 2.9 & 48.8 & 0.049 & 0.67 & 0.09 & 0.08 & 0.0001 & 0.4297 \\
\hline & GH1015 & 0.4 & 29.0 & 0.063 & 0.40 & 0.09 & 0.10 & 0.0015 & 0.3465 \\
\hline & GH1016 & 0.1 & 18.3 & 0.206 & 0.25 & 0.13 & 0.22 & 0.6081 & 0.3117 \\
\hline & GH1020 & 0.3 & 22.3 & 0.448 & 0.30 & 0.19 & 0.31 & 0.4555 & 0.2806 \\
\hline & GH1021 & 3.1 & 51.0 & 0.042 & 0.70 & 0.09 & 0.07 & 0.1173 & 0.4118 \\
\hline & $\mathrm{HN}$ & 2.9 & 46.5 & 0.095 & 0.64 & 0.13 & 0.11 & 0.0676 & 0.5883 \\
\hline & Ls16 & 0.0 & 11.5 & 0.014 & 0.15 & 0.04 & 0.05 & $\ldots$ & 0.1579 \\
\hline & Ls17 & 1.7 & 41.3 & 0.094 & 0.57 & 0.12 & 0.12 & 0.1570 & $\ldots$ \\
\hline & XJ2008 & 2.9 & 46.5 & 0.075 & 0.64 & 0.12 & 0.10 & 0.0676 & 0.5883 \\
\hline & XJ592 & 4.4 & 58.3 & 0.027 & 0.80 & 0.08 & 0.06 & 0.0125 & 0.1893 \\
\hline & Water & 0.0 & 11.5 & 0.014 & 0.15 & 0.04 & 0.05 & $\ldots$ & $\ldots$ \\
\hline
\end{tabular}

\footnotetext{
${ }^{\text {a }} \mathrm{NC}=$ not calculated
} 
previously thought. Virulence assays of these isolates on the three cultivars evaluated in this study may clarify this ambivalent characterization by the molecular markers. Thus, further study using more isolates from diverse geographic areas should be tested in the future. For the most part, however, the correspondence between the defoliating phenotypes and races was at least $90 \%$. Again, virulence assays of recalcitrant isolates characterized as neither race 1 or 2 by the markers may determine whether they are even pathogenic on cotton. The PCR assays to characterize nondefoliating or defoliating pathotypes and races simultaneously will help assess the population biology of $V$. dahliae in cotton and further aid in the development of management strategies to control this disease. Variation in race of $V$. dahliae isolates from cotton has been a controversial topic but less so the characterization of isolates as defoliating and nondefoliating pathotypes (Bejarano-Alcázar et al. 1995; Daayf et al. 1995; Korolev et al. 2008; Schnathorst and Mathre 1966). Although the three cotton cultivars FM 2484B2F, 98M-2983, and CA4002 serve to differentiate defoliating isolates from nondefoliating isolates, the race-specific PCR assays help link races to the defoliation phenotypes. The strong association between the defoliating type and race $2(97.2 \%)$ and between the nondefoliating type and race 1 $(90.8 \%)$ supports the previous speculation that race 1 of $V$. dahliae is probably the nondefoliating type in cotton (Gao et al. 2011). These results will contribute to monitoring the spread of cotton-adapted strains of $V$. dahliae, and help develop resistance in cotton cultivars to $V$. dahliae.

Table 3. Comparison of each strain with Ls16 and Ls17 using nonparametric analysis of the LD_CI and F1_LD_F1 Macro methods

\begin{tabular}{llcccc}
\hline & & & & \multicolumn{2}{c}{$P$ value } \\
\cline { 5 - 6 } Strain & PCR $^{\mathbf{a}}$ & RME $^{\mathbf{b}}$ & Variance & Ls16 & Ls17 \\
\hline GH1005 & D/Race 2 & 0.66 & 0.441 & 0.0640 & 0.3720 \\
GH1015 & ND/Race 1 & 0.53 & 1.088 & 0.5610 & 0.0890 \\
GH1016 & ND/Race 1 & 0.36 & 0.793 & 0.4180 & 0.0760 \\
GH1020 & ND/Race 1 & 0.45 & 0.853 & 0.6440 & 0.2200 \\
GH1021 & D/Race 2 & 0.67 & 0.985 & 0.1070 & 0.7460 \\
HN & D/Race 2 & 0.66 & 0.401 & 0.0160 & 0.1910 \\
Ls16 & ND/Race 1 & 0.35 & 1.033 & $\ldots$ & 0.0120 \\
Ls17 & D/Race 2 & 0.48 & 0.875 & 0.0120 & $\ldots$ \\
XJ2008 & D/Race 2 & 0.56 & 0.372 & 0.0001 & 0.1480 \\
XJ592 & D/Race 2 & 0.69 & 1.101 & 0.0050 & 0.7180 \\
Water & $\ldots$ & 0.15 & 0.0389 & 0.0001 & 0.0001 \\
\hline
\end{tabular}

${ }^{a}$ Determined by polymerase chain reaction $(\mathrm{PCR}) ; \mathrm{D}=$ defoliating isolate and $\mathrm{ND}=$ nondefoliating isolate.

b Relative marginal effect.

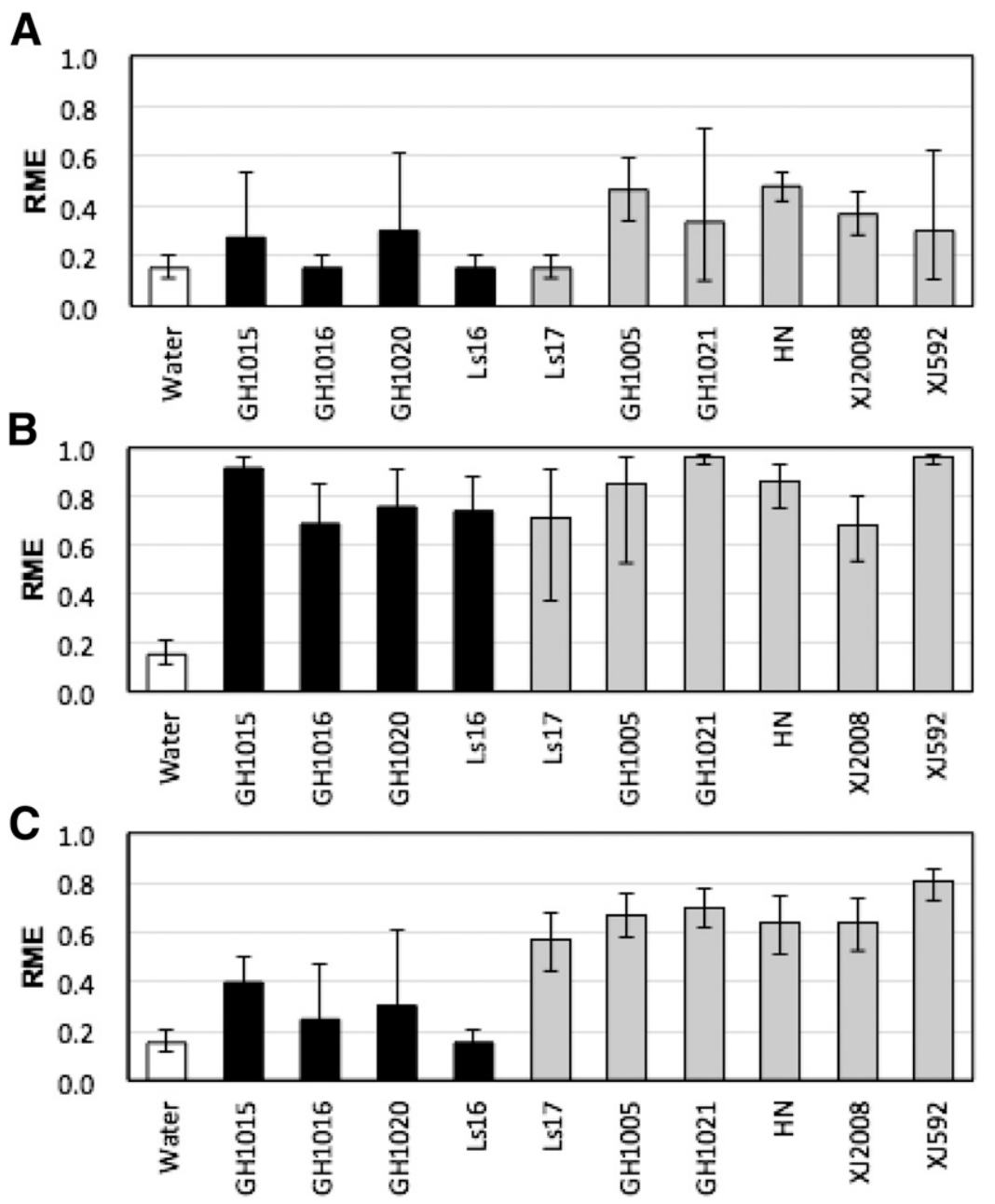

Fig. 5. Estimated relative marginal effects (RME) based on the analysis of variance-type statistics of ranked data for the severity of Verticillium wilt on $\mathbf{A}$, resistant cotton FM 2484B2F; B, susceptible cotton 98M-2983; and C, partially resistant CA4002 caused by Verticillium dahliae strains. Results represent data from two independent experiments. Confidence intervals $(95 \%)$ for relative marginal effects are represented by vertical lines. White bars represent the plants that were inoculated with sterile distilled water as a negative control. Black bars represent the plants that were inoculated with strains that were identified as nondefoliating and race 1 through polymerase chain reaction (PCR) using specific primer pairs. Gray bars represent the plants that were inoculated with strains which were identified as defoliating and race 2 through PCR using specific primer pairs. 


\section{Acknowledgments}

The study was supported partially by NSFC (31371888) and the 111 project from Education Ministry of China (number B07049) and Hatch Funds allocated to the University of California, Davis. We thank H. Q. Zhu for providing isolates for this study and $\mathrm{T}$ A. Wheeler for providing cotton cultivars evaluated in this study.

\section{Literature Cited}

Alexander, L. J. 1962. Susceptibility of certain Verticillium-resistant tomato varieties to an Ohio isolate of pathogen. Phytopathology 52:998-1000.

Bejarano-Alcázar, J., Melero-Vara, J. M., Blanco-López, M. A., and Jiménez-Díaz, R. M. 1995. Influence of inoculum density of defoliating and nondefoliating pathotypes of Verticillium dahliae on epidemics of Verticillium wilt of cotton in southern Spain. Phytopathology 85:1474-1481.

Bell, A. 1992. Verticillium wilt. Pages 87-126 in: Cotton Disease. R. J. Hillocks, ed. CAB International, Oxon.

Bhat, R. G., and Subbarao, K. V. 1999. Host range specificity in Verticillium dahliae. Phytopathology 89:1218-1225.

Carder, J. H., and Barbara, D. J. 1994. Molecular variation within some Japanese isolates of Verticillium dahliae. Plant Pathol. 43:947-950.

Chai, Y., Zhao, L., Liao, Z., Xun, X., Zuo, K., Zhang, L., Wang, S., and Tang, K. 2003. Molecular cloning of a potential Verticillium dahliae resistance gene SlVe 1 with multi-site polyadenylation from Solanum licopersicoides. Mitochondrial DNA 14:375-384.

Chen, Z. J., Scheffler, B. E., Dennis, E., Triplett, B. A., Zhang, T., Guo, W., Chen, X., Stelly, D. M., Rabinowicz, P. D., Town, C. D., Arioli, T., Brubaker, C., Cantrell, R. G., Lacape, J.-M., Ulloa, M., Chee, P., Gingle, A. R., Haigler, C. H., Percy, R., Saha, S., Wilkins, T., Wright, R. J., Deynze, A. V., Zhu, Y., Yu, S., Abdurakhmonov, I., Katageri, I., Kumar, P. A., Mehboob-ur-Rahman, Zafar, Y., Yu, J. Z., Kohel, R. J., Wendel, J. F., and Paterson, A. H. 2007. Toward sequencing cotton (Gossypium) genomes. Plant Physiol. 145:1303-1310.

Daayf, F., Nicole, M., and Geiger, J. P. 1995. Differentiation of Verticillium dahliae populations on the basis of vegetative compatibility and pathogenicity on cotton. Eur. J. Plant Pathol. 101:69-79.

de Jonge, R., van Esse, H. P., Maruthachalam, K., Bolton, M. D., Santhanam, P., Saber, M. K., Zhang, Z., Usami, T., Lievens, B., Subbarao, K. V., and Thomma, B. P. 2012. Tomato immune receptor $V e 1$ recognizes effector of multiple fungal pathogens uncovered by genome and RNA sequencing. Proc. Nat. Acad. Sci. 109:5110-5115.

Dever, J. K., Wheeler, T. A., and Kelly, C. M. 2013. Registration of CA 4002 cotton germplasm line partially resistant to Verticillium wilt. J. Plant Reg. 7:209-215.

Fei, J., Chai, Y., Wang, J., Lin, J., Sun, X., Sun, C., Zuo, K., and Tang, K. 2004. cDNA cloning and characterization of the Ve homologue gene StVe from Solanum torvum Swartz. Mitochondrial DNA 15:88-95.

Fradin, E. F., Abd-El-Haliem, A., Masini, L., van den Berg, G. C. M., Joosten, M. H. A. J., and Thomma, B. P. H. J. 2011. Interfamily transfer of tomato Vel mediates Verticillium resistance in Arabidopsis. Plant Physiol. 156:2255-2265.

Fradin, E. F., Zhang, Z., Ayala, J. C., Castroverde, C. D., Nazar, R. N., Robb, J., Liu, C. M., and Thomma, B. P. 2009. Genetic dissection of Verticillium wilt resistance mediated by tomato Ve1. Plant Physiol. 150:320-332.

Friebertshauser, G., and DeVay, J. 1982. Differential effects of the defoliating and nondefoliating pathotypes of Verticillium dahliae upon the growth and development of Gossypium hirsutum. Phytopathology 72:872-877.

Gao, X., Wheeler, T., Li, Z., Kenerley, C. M., He, P., and Shan, L. 2011. Silencing GhNDR1 and GhMKK2 compromises cotton resistance to Verticillium wilt. Plant J. 66:293-305.

Grogan, R., Ioannou, N., Schneider, R. W., Sall, M. A., and Kimble, K. A. 1979. Verticillium wilt on resistant tomato cultivars in California: Virulence of isolates from plants and soils and relationship of inoculum density to disease incidence. Phytopathology 69:1176-1180.

Gurung, S., Short, D. P. G., Atallah, Z. K., and Subbarao, K. V. 2014. Clonal expansion of Verticillium dahliae in lettuce. Phytopathology 104:641-649.

Hayes, R., McHale, L. K., Vallad, G. E., Truco, M. J., Michelmore, R. W., Klosterman, S. J., Maruthachalam, K., and Subbarao, K. V. 2011. The inheritance of resistance to Verticillium wilt caused by race 1 isolates of Verticillium dahliae in lettuce cultivar La Brillante. Theor. Appl. Genet. 123:509-517.

Heinz, R., Lee, S., Saparno, A., Nazar, R., and Robb, J. 1998. Cyclical systemic colonization in Verticillium-infected tomato. Physiol. Mol. Plant Pathol. 52:385-396.

Hu, D. F., Wang, C. S., Tao, F., Cui, Q., Xu, X. M., Shang, W. J., and Hu, X. P. 2014. Whole genome wide expression profiles on germination of Verticillium dahliae microsclerotia. PLoS One 9:e100046.

Inderbitzin, P., Davis, R. M., Bostock, R. M., and Subbarao, K. V. 2013. Identification and differentiation of Verticillium species and V. longisporum lineages by simplex and multiplex PCR assays. PLoS One 8:e65990.

Inderbitzin, P., and Subbarao, K. V. 2014. Verticillium systematics and evolution: How confusion impedes Verticillium wilt management and how to resolve it. Phytopathology 104:564-574.

Jansky, S. H., Rouse, D. I., and Kauth, P. J. 2004. Inheritance of resistance to Verticillium dahliae in diploid interspecific potato hybrids. Plant Dis. 88:1075-1078.

Joaquim, T. R., and Rowe, R. C. 1990. Reassessment of vegetative compatibility relationships among strains of Verticillium dahliae using nitrate-nonutilizing mutants. Phytopathology 80:1160-1166.
Kabir, Z., Bhat, R., and Subbarao, K. 2004. Comparison of media for recovery of Verticillium dahliae from soil. Plant Dis. 88:49-55.

Kawchuk, L. M., Hachey, J., Lynch, D. R., Kulcsar, F., van Rooijen, G., Waterer, D. R., Robertson, A., Kokko, E., Byers, R., Howard, R. J., Fischer, R., and Prufer, D. 2001. Tomato Ve disease resistance genes encode cell surface-like receptors. Proc. Natl. Acad. Sci. USA 98:6511-6515.

Klosterman, S. J., Atallah, Z. K., Vallad, G. E., and Subbarao, K. V. 2009 Diversity, pathogenicity, and management of Verticillium species. Annu. Rev. Phytopathol. 47:39-62.

Korolev, N., Pérez-Artés, E., Mercado-Blanco, J., Bejarano-Alcázar, J., Rodríguez-Jurado, D., Jiménez-Díaz, R. M., Katan, T., and Katan, J. 2008. Vegetative compatibility of cotton-defoliating Verticillium dahliae in Israel and its pathogenicity to various crop plants. Eur. J. Plant Pathol. 122:603-617.

Lu, W. J., Liu, Y. J., Zhu, H. Q., Shang, W. J., Yang, J. R., and Hu, X. P. 2013. Verticillium wilt of redbud in China caused by Verticillium dahliae. Plant Dis. 97:1513.

Maruthachalam, K., Atallah, Z. K., Vallad, G. E., Klosterman, S. J., Hayes, R. J., Davis, R. M., and Subbarao, K. V. 2010. Molecular variation among isolates of Verticillium dahliae and polymerase chain reaction-based differentiation of races. Phytopathology 100:1222-1230.

Nachmias, A., Buchner, V., and Burstein, Y. 1985. Biological and immunochemical characterization of a low molecular weight phytotoxin isolated from a proteinlipopolysaccharide complex produced by a potato isolate of Verticillium dahliae Kleb. Physiol. Plant Pathol. 26:43-55.

Okoli, C. A. N., Carder, J. H., and Barbara, D. J. 1993. Molecular variation and sub-specific groupings within Verticillium dahliae. Mycol. Res. 97:233-239.

Pegg, G. F., and Brady, B. L. 2002. Verticillium Wilts. CABI Publishing, New York

Pérez-Artés, E., García-Pedrajas, M. D., Bejarano-Alcázar, J., and Jiménez-Díaz, R. M. 2000. Differentiation of cotton-defoliating and nondefoliating pathotypes of Verticillium dahliae by RAPD and specific PCR analyses. Eur. J. Plant Pathol. 106:507-517.

Popov, V. I., Tarunina, T. A., and Usmanov, Z. U. 1972. On the physiologic races of Verticillium dahliae Kleb., a causal agent of cotton wilt. Mikol. Fitopatol. 6:500-502

Portenko, L. G., and Kas'yanenko, A. G. 1987. Genetics of the cotton wilt pathogen Verticillium dahliae Kleb for virulence and aggressiveness. Genet. USSR 23:1859-1865.

Puhalla, J. E., and Hummel, M. 1983. Vegetative compatibility groups within Verticillium dahliae. Phytopathology 73:1305-1308.

Ramsay, J. R., Multani, D. S., and Lyon, B. R. 1996. RAPD-PCR identification of Verticillium dahliae isolates with differential pathogenicity on cotton. Crop Pasture Sci. 47:681-693.

Schnathorst, W. C., and Mathre, D. E. 1966. Host range and differentiation of a severe form of Verticillium albo-atrum in cotton. Phytopathology 56:1156-1161.

Schnathorst, W. C., and Sibbett, G. S. 1971. The relation of strains of Verticillium albo-atrum to severity of Verticillium wilt in Gossypium hirsutum and Olea europea in California. Plant Dis. Rep. 55:780-782.

Shah, D., and Madden, L. 2004. Nonparametric analysis of ordinal data in designed factorial experiments. Phytopathology 41:986-990.

Short, D. P., Gurung, S., Maruthachalam, K., Atallah, Z., and Subbarao, K. V. 2014. Verticillium dahliae race 2-specific PCR reveals a high frequency of race 2 strains in commercial spinach seed lots and delineates race structure. Phytopathology 104:779-785.

Subbarao, K. V., Hubbard, J. C., Greathead, A. S., and Spencer, G. A. 1997. Verticillium wilt. Pages 26-27 in: Compendium of Lettuce Diseases. R. M. Davis, K. V. Subbarao, R. N. Raid, and E. A. Kurtz, eds. The American Phytopathological Society, St. Paul, MN.

Tjamos, E. 1981. Virulence of Verticillium dahliae and V. albo-atrum isolates in tomato seedlings in relation to their host of origin and the applied cropping system. Phytopathology 71:98-100.

Vallad, G. E., Qin, Q. M., Grube, R., Hayes, R. J., and Subbarao, K. V. 2006. Characterization of race-specific interactions among isolates of Verticillium dahliae pathogenic on lettuce. Phytopathology 96:1380-1387.

Vining, K. J., Zhang, Q., Smith, C., and Davis, T. 2007. Identification of resistance gene analogs and Verticillium wilt resistance-like sequences in Mentha longifolia. J. Am. Soc. Hortic. Sci. 132:541-550.

Wilhelm, S. 1955. Longevity of the Verticillium wilt fungus in the laboratory and field. Phytopathology 45:180-181.

Xia, Z., Achar, P. N., and Gu, B. 1998. Vegetative compatibility groupings of Verticillium dahliae from cotton in mainland China. Eur. J. Plant Pathol. 104:871-876.

Zhang, B., Yang, Y., Chen, T., Yu, W., Liu, T., Li, H., Fan, X., Ren, Y., Shen, D. Liu, L., Dou, D., and Chang, Y. 2012. Island cotton Gbvel gene encoding a receptor-like protein confers resistance to both defoliating and nondefoliating isolates of Verticillium dahliae. PLoS One 7:e51091.

Zhang, Y., Wang, X., Yang, S., Chi, J., Zhang, G., and Ma, Z. 2011. Cloning and characterization of a Verticillium wilt resistance gene from Gossypium barbadense and functional analysis in Arabidopsis thaliana. Plant Cell Rep. 30:2085-2096.

Zhu, Y. Y., Xu, Q. J., Lei, J., Yang, J. R., and Hu, X. P. 2012. Identification of defoliating and nondefoliating pathotype of Verticillium dahliae isolated from cotton in Shaanxi. Acta Agric. Boreali-Occidentalis Sin. 21:166-73 (In Chinese with English abstract). 


\section{ERRATUM / Volume 99, Number 12, 2015 / PDIS-03-15-0261-RE}

In the article "Nondefoliating and Defoliating Strains from Cotton Correlate with Races 1 and 2 of Verticillium dahliae" by X.-P. Hu, S. Gurung, D. P. G. Short, G. V. Sandoya, W.-J. Shang, R. J. Hayes, R. M. Davis, and K. V. Subbarao, figure 1 had to be replaced due to poor quality DNA extracted from some isolates. The extractions were performed again and the figure shown below is now correct.

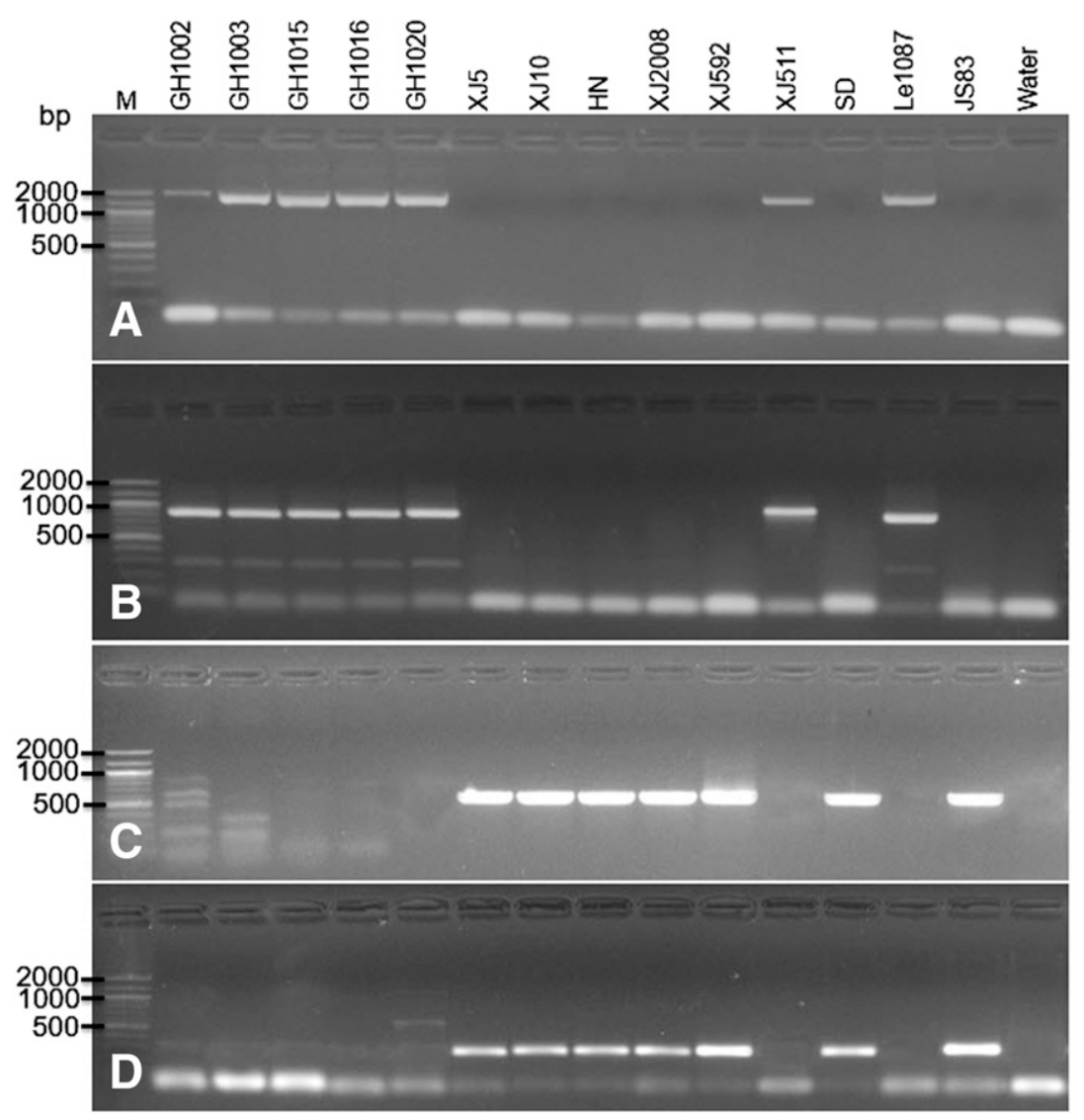

Fig. 1. Agarose gel pattern of PCR amplicons generated with defoliating, nondefoliating, and race 1- and race 2-specific primer pairs of Verticillium dahliae. A, Amplicons generated with the nondefoliating-specific primers NDF and NDR. B, Amplicons generated with the race 1-specific primers VdAve1F and VdAve1R. C, Amplicons generated with the defoliating-specific primers DF and DR. D, Amplicons generated with the race 2-specific primers VdR2F and VdR2R. The 1.5\% (w/v) agarose gel was used. Lane $\mathrm{M}=$ 2,000-bp DNA ladder used as a size marker. Strains GH1002, GH1003, GH1015, GH1016, and GH1020 collected from cotton, California; strain HN collected from cotton, Henan, China; strains XJ5, XJ10, XJ2008, XJ592, and XJ511 collected from cotton, Xinjiang, China; strain SD collected from cotton in Shandong, China; strain JS83 collected from cotton, Jiangsu, China; strain Le1087 collected from tomato, California. Sterile distilled water was used as a negative control. 